\title{
Robust Simulation Methodology for Surface-Roughness Loss in Interconnect and Package Modelings
}

\author{
Quan Chen, Student Member, IEEE, Hoi Wai Choi, Senior Member, IEEE, and Ngai Wong, Member, IEEE
}

\begin{abstract}
In multigigahertz integrated-circuit design, the extra energy loss caused by conductor surface roughness in metallic interconnects and packagings is more evident than ever before and demands explicit consideration for accurate prediction of signal integrity and energy consumption. Existing techniques based on analytical approximation, despite simple formulations, suffer from restrictive valid ranges, namely, either small or large roughness/frequencies. In this paper, we propose a robust and efficient numerical-simulation methodology applicable to evaluating general surface roughness, described by parameterized stochastic processes, across a wide frequency band. Traditional computation-intensive electromagnetic simulation is avoided via a tailored scalar-wave modeling to capture the power loss due to surface roughness. The spectral stochastic collocation method is applied to construct the complete statistical model. Comparisons with full wave simulation as well as existing methods in their respective valid ranges then verify the effectiveness of the proposed approach.
\end{abstract}

Index Terms-Interconnect, power loss, rough surface, scalar-wave modeling (SWM), spectral stochastic collocation.

\section{INTRODUCTION}

$\mathbf{S}$ URFACE ROUGHNESS in metal conductors of interconnects and microelectronic packagings is sometimes due to process variations in fabrication and, more frequently, artificial roughening processes, e.g., electronic deposition and chemical etching, to promote interfacial adhesion between dielectrics and conducting materials. Fig. 1(a) shows the cross section of a metal trace with roughness around it, while Fig. 1(b) shows an atomic-force microscopy (AFM) for the top view of a printed circuit board (PCB) interconnect demonstrating the 3-D rough surface. It has been reported by measurements that the surface-profile variation in roughened copper foil can cause an increase of resistivity by a factor of two to three in microwave frequencies [1]. Recent experimental studies can be found in [2] and [3]. Such impact on resistivity breaks the predictive capacity of classical high-frequency relationship

Manuscript received January 30, 2009; revised April 23, 2009, June 8, 2009, and July 23, 2009. Current version published October 21, 2009. This work was supported in part by the Hong Kong Research Grants Council under Project HKU 717407E and in part by the University Research Committee of The University of Hong Kong. This paper was recommended by Associate Editor P. Li.

The authors are with the Department of Electrical and Electronic Engineering, The University of Hong Kong, Pokfulam Road, Hong Kong (e-mail: quanchen@eee.hku.hk; hwchoi@eee.hku.hk; nwong@eee.hku.hk).

Color versions of one or more of the figures in this paper are available online at http://ieeexplore.iee.org.

Digital Object Identifier 10.1109/TCAD.2009.2030408
$R_{f} \propto \sqrt{f}$ for the frequency-dependent resistance $R_{f}$. With the ever-increasing signaling rate, particularly in offchip communications of multiprocessor architectures, an accurate estimation of rough-surface effect is imperative for improved prediction of insertion loss, signal integrity, and thermomechanical reliability in interconnect-aware design methodologies and tools.

Attempts to evaluate the roughness-induced loss by incorporating surface variations directly into specified field solvers have been made, e.g., in [4]. However, the computation is highly expensive due to the huge problem size resulting from numerical simulation with surfaces of the whole structure. A more viable way is to scale the conducting loss by a frequencydependent factor that quantifies the local impact of surface roughness, which can as well be used in ordinary simulators to scale the reference values of resistance and inductance extracted from smooth-surface conductor to generate more accurate simulation models. In addition, the current-density-independent loss-scaling factor can be reused for different interconnect structures with similar rough-surface characteristics, e.g., those produced by the same processing techniques.

Most existing techniques simply calculate this factor in a closed-form manner. For instance, the guideline model in industrial practice, Hammerstad formula, estimates the loss-scaling factor for 2-D rugged structures by [5]

$$
\frac{P_{r}}{P_{s}}=\left[1+\frac{2}{\pi} \tan ^{-1}\left(1.4\left(\frac{\sigma}{\delta}\right)^{2}\right)\right]
$$

where $P_{r}$ and $P_{s}$ denote the power loss of a rough-surface conductor and its smooth-surface counterpart, respectively. Here, $\sigma$ is the standard deviation of surface height, $\delta=\sqrt{\rho /(\pi f \mu)}$ is the skin depth, $\rho$ is the conductor resistivity, and $\mu$ is the freespace permeability. Since only $\sigma$ of the surface characteristics is considered, (1) is subjected to frequent modifications to match field-measurement data from different environments where surface roughness depends on more than a single parameter.

More practical models for 3-D surface roughness include the second-order small perturbation method (SPM2) [6] (which models the surface roughness by stochastic processes) and the hemispherical boss modeling (HBM) [7] (which models surface irregularities as size-controlled hemispherical bosses distributed, regularly or randomly, on a flat plane). Nevertheless, the efficiency gain of closed-form models comes at the cost of applicability, namely, both SPM2 and HBM suffer from restricted valid regions. More specifically, SPM2 requires the root mean-square (rms) height of roughness, and the surface 


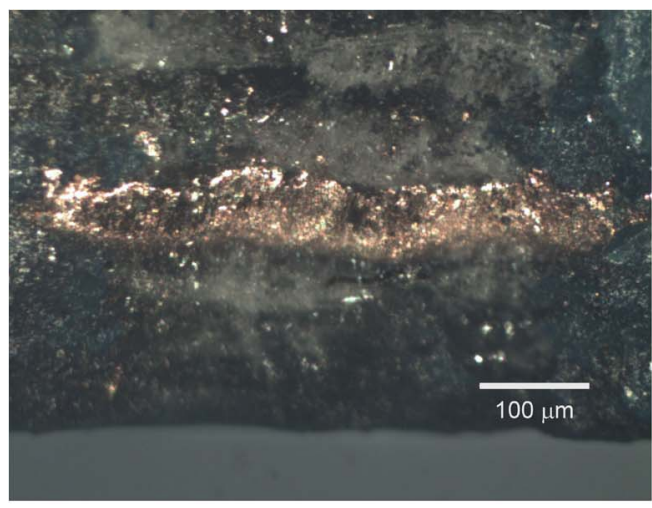

(a)

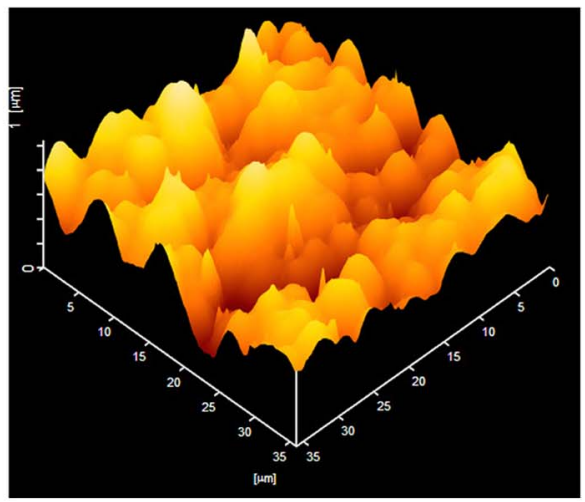

(b)

Fig. 1. (a) Micrograph of the cross section of a metal trace. (b) Top view of a 3-D rough surface in a PCB interconnect scanned by AFM
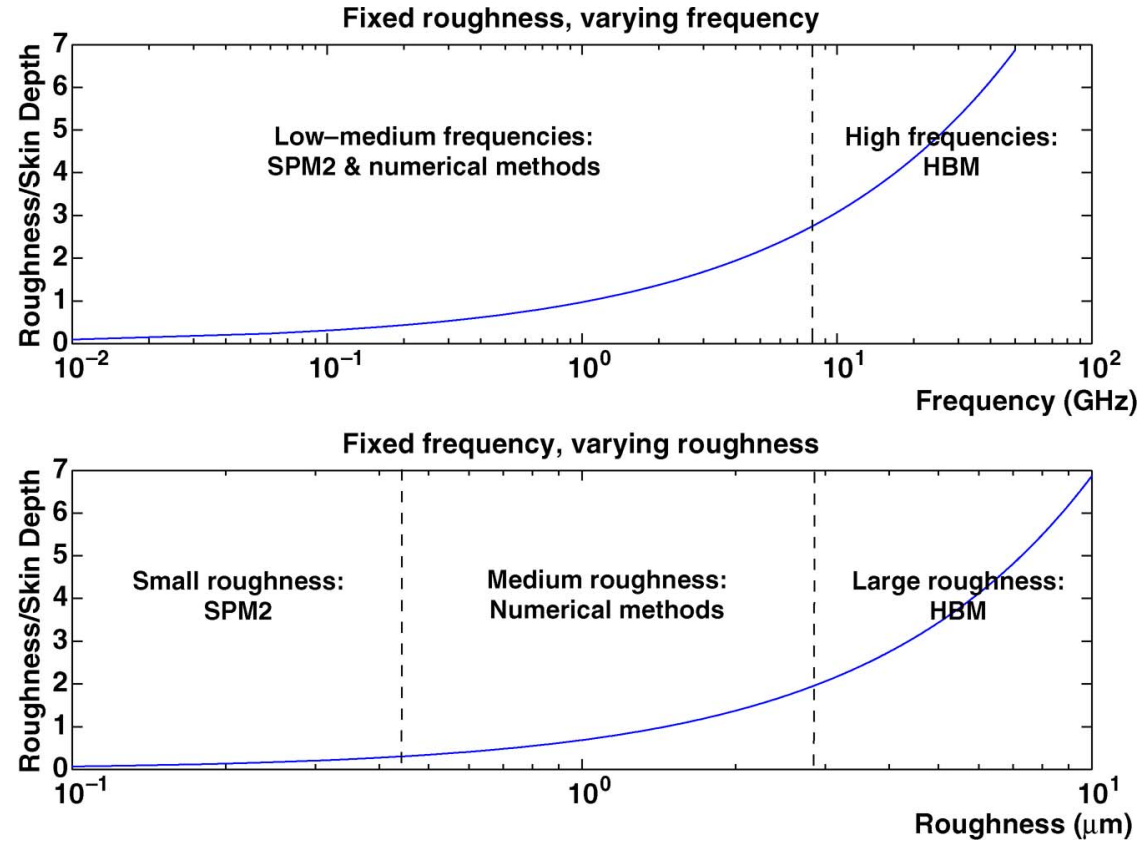

Fig. 2. Valid regions of SPM2 and HBM w.r.t. the ratio of roughness to skin depth.

slope are small to validate the presumption of "perturbation," which limits its applicability to small roughness and low-tomedium clock frequencies. To the opposite of SPM2, HBM primarily works under the scenarios with large roughness or very high frequencies, where the skin depth is small compared with the radii of the bosses. Such restrictiveness demands a careful selection of proper closed-form techniques for given scenarios, and undesirable, or even impossible, model switches if simulation domain exceeds the valid region of a single technique. Fig. 2 roughly shows the corresponding valid regions of SPM2 and HBM w.r.t. the ratio of roughness and skin depth for fixed roughness with varying and fixed frequencies with varying roughness, although, in general, there is not a solid boundary for one's valid region and the validity of a given model has to be judged within the context. Consequently, the figure suggests that there is still a lack of efficient simulation methods for evaluating surface-roughness loss at the intermediate frequency/roughness ranges where the skin depth has the same order of dimension as the roughness, which is often the case in practice.

Furthermore, surface roughness is usually regarded as a source of variation in stochastic analysis of interconnect and packaging simulation. However, the closed-form techniques can only calculate the mean value of loss-scaling factor and cannot be used to compute the standard deviation or complete distribution of the factor. This makes it difficult, if not impossible, to generate models compatible with postprocessing stochastic-model order reduction and statistical timinganalysis tools [8], [9], most of which require the availability of high-order statistics. A numerical-evaluation approach of lossscaling factor allowing the acquisition of second-order variance has been proposed by [10] using direct stochastic integral equation (IE). However, the technique is only applicable to 2-D surface model assuming a constant magnetic field on the surface, which largely restricts its accuracy, since practical problems are generally 3-D with dynamic magnetic field. 
To address these problems, namely, lack of generally valid higher order statistical models, we employ a numerically robust methodology, called scalar-wave modeling (SWM), to simulate the impact of 3-D surface roughness with diverse roughness patterns over a wide frequency range. While the preliminary idea of SWM for rough-surface loss analysis was first proposed in [11] by us, it is further developed into a more accurate and usable form utilizing triangular discretization of rough surfaces. New results are presented regarding the limitations of SPM2 and HBM, and comparison against electromagnetic (EM) vectorial modeling confirms the efficacy of the SWM approach. In particular, in SWM, a rough surface is modeled by a parameterized stochastic process. The complicated EM problem is transformed into a simplified scalar representation by regarding the EM wave as a scalar energy flux. In this way, the extra loss caused by surface roughness can be approximated by the amount of energy absorbed by the rough surface upon which the scalar flux is incident. The surface IEs thus established are numerically solved by the method of moments (MOM). An efficient stochastic solver, spectral stochastic collocation method (SSCM) [12], is applied to generate the second-order statistical model of the loss-scaling factor so that conventional (expensive) Monte Carlo (MC) simulations can be avoided.

This paper is organized as follows. Section II reviews the characterization of a 3-D random rough surface via stationary stochastic processes and the formulas of SPM2 and HBM. Formulation and solution of the proposed SWM are detailed in Section III. Verification against vectorial EM modeling is given in the same section. Numerical comparisons with existing techniques in their respective valid regions are then conducted in Section IV to verify the effectiveness of SWM. Finally, Section V draws the conclusion.

\section{BACKGROUND REVIEW}

For the 3-D random rough surface of a conductor, we describe the surface height $h\left(\bar{r}_{\perp}\right)$ as a stationary stochastic process, where $\bar{r}_{\perp}=(x, y)$ falls on the mean plane. A stochastic process is generally characterized by the probability density function (PDF) and the spatial correlation function (CF) $C\left(\bar{r}_{\perp i}, \bar{r}_{\perp j}\right)$. Without loss of generality, we use the Gaussian PDF

$$
\operatorname{PDF}\left(h\left(\bar{r}_{\perp}\right)\right)=\frac{1}{\sqrt{2 \pi} \sigma} \exp \left(-\frac{h^{2}\left(\bar{r}_{\perp}\right)}{2 \sigma^{2}}\right)
$$

in this paper associated with different CFs. Here, $\sigma$ is the standard deviation, and the mean plane is on $h=0$.

An important feature of this characterization is that the stochastic process parameters, e.g., $\sigma$ and $C$, can be quantitatively extracted from a real interconnect surface by measuring surface height as a function of position [13]. Subsequently, different surface roughness, in reality, can be reproduced and simulated by properly parameterizing the stochastic processes. Fig. 3 shows a simulated 3-D random rough surface with the common Gaussian CF, $C(d)=\sigma^{2} \exp \left(-d^{2} / \eta^{2}\right)$, where $d=\left|\bar{r}_{\perp i}-\bar{r}_{\perp j}\right|$ and $\eta$ is the correlation length. In general, a larger $\sigma$ or a smaller $\eta$ implies a rougher surface.

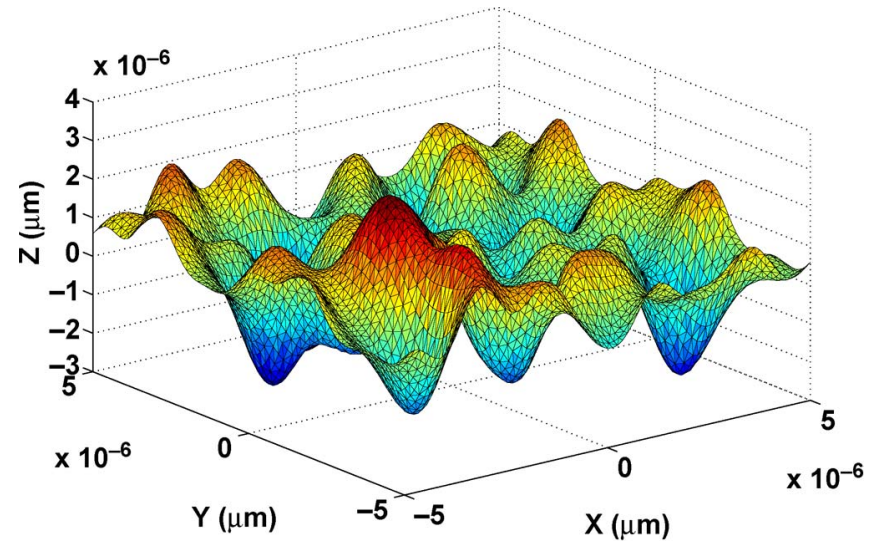

Fig. 3. Simulated 3-D random rough surface with Gaussian CF and $\sigma=\eta=1 \mu \mathrm{m}$.

The closed-form SPM2 and HBM techniques are succinctly reviewed. On one hand, SPM2 [6] assumes small surface height and slope, and uses the aforementioned stochastic surface modeling for calculating the mean value of loss-scaling factor by

$\frac{P_{r}}{P_{s}}=1+\frac{2 \sigma^{2}}{\delta^{2}}-\frac{2}{\delta} \iint d k_{x} d k_{y} W\left(k_{x}, k_{y}\right) \operatorname{Re} \sqrt{\frac{2 j}{\delta^{2}}-k_{x}^{2}-k_{y}^{2}}$

where the spectral density function $W\left(k_{x}, k_{y}\right)$ is the Fourier transform of the CF. On the other hand, HBM [7] models surface roughness as conducting protrusions and calculates the loss-scaling factor by

$$
\frac{P_{r}}{P_{s}}=\frac{\left|\operatorname{Re}\left[\Omega \frac{3 \pi}{4 k_{1}^{2}}(\alpha(1)+\beta(1))\right]\right|+\frac{\mu \omega \delta}{4}\left(A_{\text {tile }}-A_{\text {base }}\right)}{\frac{\mu \omega \delta}{4} A_{\text {tile }}}
$$

where $\omega=2 \pi f, k_{1}=\omega \sqrt{\mu \epsilon_{1}}$ is the wavenumber, and $\Omega=$ $\sqrt{\mu / \epsilon_{1}}\left(\epsilon_{1}\right.$ being the permittivity) denotes the wave impedance of the upper medium. Here, $\alpha(1)$ and $\beta(1)$ are the approximated scattering coefficients which can be analytically evaluated only when $\delta$ is small compared with the size of the protrusion. $A_{\mathrm{tile}}$ and $A_{\text {base }}$ are the tile areas of the plane surrounding the protrusion and the base area of the hemisphere with equal volume to the protrusion, respectively.

SPM2 generally breaks down as the dimensions of roughness increase, particularly, the slope of rough surface becomes large. HBM is a gross assumption as surface roughness in real interconnects is substantially diverse, which is difficult to be modeled by simple bosses (see Section IV where numerical examples demonstrate the inadequacy of the hemispherical modeling in HBM). Moreover, HBM becomes invalid when $\delta$ is close to or greater than the height of protrusion, since, in this case, the energy dissipated by a protrusion is even smaller than that by a flat plane with an area equal to the base of the protrusion [7]. Numerical examples are given in Section IV to confirm the universal applicability of the proposed SWM approach versus the restrictive natures of SPM2 and HBM. 


\section{SWM}

In principle, EM wave theory can be used to rigorously determine the field scattered or absorbed by a conducting body, which has been extensively studied for decades [14]. Direct application of numerical EM simulation to predict surfaceroughness loss in microscopic interconnects, however, faces the following difficulties.

1) The wavelength in dielectrics (on the order of centimeters) is large compared with the dimension of a conductor surface (on the order of micrometers). This corresponds to the well-known low-frequency problem in traditional EM scattering from metallic objects, in which the numerical solution of IE can become unstable due to the dominant impact of hypersingular integral operator [15]. Remedies of such problem include using high-order loop star basis or modifying the integral operators, all of which will significantly increase the complexity of calculation.

2) The computation is intensive if direct EM formulation is applied due to its vectorial nature, particularly when a fine discretization of the surface is needed to properly capture the details of surface variation. The situation becomes worse if the low-frequency problem (requires higher order basis or operator) and random surface modeling (requires multiple sampling) are taken into account.

To balance the cost and performance, we choose scalar-wave theory instead of EM wave theory to formulate the energy loss caused by surface roughness in interconnects and packagings. SWM has long been used as a simplifying substitute for vectorial EM wave to predict wave behavior and observe important phenomena in scattering problems [16]. It has been shown that the results of SWM are well correlated to that from EM wave modeling in many cases, particularly when the detailed field distribution is not required [17].

To formulate the problem with SWM, we consider a general two-medium case, where the upper half-space of a rough interface $S$ is filled by dielectrics (medium 1) with electrical parameters $\epsilon_{1}, \mu$, and wavenumber $k_{1}$ as in (4), and the lower half by conducting material (medium 2) with $\rho, \mu$, and $k_{2}=(1+j) / \delta$. One considers a scalar plane wave normally impinging on the rough surface $\psi_{i n}(\bar{r})=\exp \left(-j k_{0} z\right)$, where $\bar{r}=(x, y, z)$; then the wave functions $\psi_{i}, i=1,2$ in both media obey the 3-D Helmholtz equation

$$
\left(\nabla^{2}+k_{i}^{2}\right) \psi_{i}=0
$$

On applying the Green's second identity, two coupled surface IEs can be established [17]

$$
\begin{aligned}
& \iint_{S} d s^{\prime} \hat{n}^{\prime} \cdot\left[G_{1}\left(\bar{r}, \bar{r}^{\prime}\right) \nabla^{\prime} \psi_{1}\left(\bar{r}^{\prime}\right)-\nabla^{\prime} G_{1}\left(\bar{r}, \bar{r}^{\prime}\right) \psi_{1}\left(\bar{r}^{\prime}\right)\right] \\
& +\psi_{1}(\bar{r})=\psi_{i n}(\bar{r}), \quad \bar{r}, \bar{r}^{\prime} \in S \\
& \iint_{S} d s^{\prime} \hat{n}^{\prime} \cdot\left[G_{2}\left(\bar{r}, \bar{r}^{\prime}\right) \nabla^{\prime} \psi_{2}\left(\bar{r}^{\prime}\right)-\nabla^{\prime} G_{2}\left(\bar{r}, \bar{r}^{\prime}\right) \psi_{2}\left(\bar{r}^{\prime}\right)\right] \\
& \quad-\psi_{2}(\bar{r})=0, \quad \bar{r}, \bar{r}^{\prime} \in S
\end{aligned}
$$

where $\hat{n}^{\prime}$ is the unit surface normal vector pointing outward at $\bar{r}^{\prime}, \nabla^{\prime}$ is the gradient w.r.t. $\bar{r}^{\prime}$, and $G_{i}, i=1,2$ is the scalar Green's function in the $i$ th medium with

$$
G_{i}\left(\bar{r}, \bar{r}^{\prime}\right)=\frac{\exp \left(j k_{i}\left|\bar{r}-\bar{r}^{\prime}\right|\right)}{4 \pi\left|\bar{r}-\bar{r}^{\prime}\right|}
$$

It should be noted that, unlike electric or magnetic field, the scalar field $\psi$ (including $\psi_{1}, \psi_{2}$, and $\psi_{i n}$ ) does not have a physical meaning and, intuitively, can be interpreted as the velocity potential of a scalar energy flux. Analogously, one can view the scalar flux as a sound wave in which $\psi$ corresponds to the sound pressure (linearly proportional to the acousticvelocity potential) [18].

The essence of our formulation is to calculate the amount of energy absorbed by a rough surface subjected to a given incident acoustics-like scalar flux, which serves as an approximation of the real EM energy absorbed by the same surface. The purpose of such transformation is to achieve a balance between the extreme efficiency of closed-form techniques and the extreme accuracy of rigorous EM simulation, leading to a tradeoff that enjoys simple implementation and fast computation while still preserving sufficient simulation capacity for generic cases.

\section{A. Continuous Boundary Condition for Scalar Waves}

To solve (6), an appropriate boundary condition must be established to relate the field unknowns in the two media. Considering our goal to approximate an EM wave by a scalar wave, this boundary condition should properly model the physical behavior of a real EM field transmitting from dielectrics into a highly conducting medium. Since conventional boundary conditions, e.g., Dirichlet $(\psi=0)$, Neuman $(\hat{n} \cdot \nabla \psi=0)$, or acoustic impedance $(a \psi+b \hat{n} \cdot \nabla \psi=0)$, are insufficient to capture the continuity of EM field across the rough boundary, a new boundary condition tailored for SWM is derived in the following by exploiting the energy equivalence between EM wave and acoustic wave as a representative of scalar wave.

As indicated in [6] and [7], the distribution of EM field near the surface of practical quasi-transverse EM interconnect structures is similar to that induced by an incident transversemagnetic wave. Since the wavelength in dielectric is much larger than the scale of surface roughness, the magnetic field $\bar{H}$ very close to the surface can be approximated by its tangential component $\bar{H}_{t}$. If we associate $\psi$ with a physical meaning as the magnitude of $\bar{H}_{t}$, the following relationship for $\bar{H}_{t}$, as well as the tangential electrical field $\bar{E}_{t}$ holds:

$$
\begin{aligned}
\bar{H} \approx \bar{H}_{t} & =\hat{t}_{H} \psi \\
\bar{E}_{t} & =\hat{t}_{E}\left[\frac{1}{j \omega \epsilon}(v \psi-\hat{n} \cdot \nabla \psi)\right]
\end{aligned}
$$

where $\hat{t}_{H}$ and $\hat{t}_{E}$ refer to the unit tangential vectors for $\bar{H}$ and $\bar{E}$, respectively, and $v=\left|\hat{n} \times \hat{n} \times\left(\nabla \times \hat{t}_{H}\right)\right|$. The detailed derivation of (9) is given in the Appendix. The validity of such association can be shown by calculating the approximated 
intensity of EM energy (Poynting vector) normal to the rough surface by

$$
\begin{aligned}
\bar{S}_{\mathrm{EM}} & \approx \hat{n} \cdot \frac{1}{2} \operatorname{Re}\left\{\bar{E}_{t} \times \bar{H}_{t}^{*}\right\} \\
& =\frac{1}{2} \operatorname{Re}\left\{\hat{n} \cdot\left(\hat{t}_{E} \times \hat{t}_{H}\right)\left[\frac{1}{j \omega \epsilon}(v \psi+\hat{n} \cdot \nabla \psi) \psi^{*}\right]\right\} \\
& =\frac{1}{2} \operatorname{Re}\left\{\frac{1}{j \omega \epsilon}\left[v \psi \psi^{*}+\psi^{*}(\hat{n} \cdot \nabla \psi)\right]\right\} \\
& =\frac{1}{2} \operatorname{Re}\left\{\frac{1}{j \omega \epsilon}\left[\psi^{*}(\hat{n} \cdot \nabla \psi)\right]\right\}
\end{aligned}
$$

and recognizing its consistency with the acoustic intensity normal to the rough surface calculated by [18]

$$
\begin{aligned}
\bar{S}_{\text {Acous }} & =\hat{n} \cdot \frac{1}{2} \operatorname{Re}\left\{p^{*} \frac{1}{j \omega \rho_{m}} \nabla p\right\} \\
& =\frac{1}{2} \operatorname{Re}\left\{\frac{1}{j \omega \rho_{m}} p^{*}(\hat{n} \cdot \nabla p)\right\}
\end{aligned}
$$

where $p$ refers to the sound pressure and $\rho_{m}$ the material density of the medium. Here, we assume $\epsilon$ is real. More specifically, it means that with the equivalence between $\psi$ and $\left|\bar{H}_{t}\right|$ at the boundary, the intensity of energy flux of an EM wave can be well approximated by that of a scalar wave. Note that the earlier derivation is only valid for regions very close to the boundary, and, for other regions, the $\bar{E}_{t}$ and $\bar{H}_{t}$ in (10) should be replaced by $\bar{E}$ and $\bar{H}$, respectively. Finally, recalling the continuity of tangential fields across the boundary $\bar{H}_{t 1}=\bar{H}_{t 2}, \bar{E}_{t 1}=\bar{E}_{t 2}$, we have the following boundary conditions for $\psi$ and $\hat{n} \cdot \nabla \psi$ :

$$
\begin{aligned}
\psi_{1}(\bar{r}) & =\psi_{2}(\bar{r}) \\
\hat{n} \cdot \nabla \psi_{1}(\bar{r}) & =(1-\gamma) v(\bar{r}) \psi_{2}(\bar{r})+\gamma \hat{n} \cdot \nabla \psi_{2}(\bar{r})
\end{aligned}
$$

where $\gamma=\epsilon_{1} / \epsilon_{2}=-j \omega \epsilon_{1} \rho$.

\section{B. Doubly Periodic Surface Assumption}

Equation (6) should be fundamentally solved on the whole ensemble of realistic conductor surfaces, which, however, is computationally prohibitive. However, significant simplification is achievable via exploiting the particular properties of rough-surface problems in interconnect and packaging simulations. There are three important physical observations.

1) The impact of surface roughness on resistive loss is local, i.e., mutual coupling between surface roughness on separate conductors or different surfaces of one conductor is negligible.

2) The roughness is globally uniform for conductor in the same layers such that a global factor of loss enhancement can be obtained from local analysis. For nonuniform (e.g., piecewise-uniform) rough structures, local loss-scaling factors can be determined and submitted to 2-D fieldsolvers to obtain self-consistent parasitic parameters [13].

3) The spatial correlation of roughness is small compared with the geometry of conductor surfaces, which means that the surface contains, for random modeling, many peaks and valleys, or for HBM, a large number of bosses. This has been confirmed by physical measurements [7].
In light of these observations, a doubly periodic condition [17] is applied to restrict the investigation domain to within a small patch of the whole surface by enforcing $f(x+p L, y+$ $q L)=f(x, y)$, where $L$ is the periodical length and $p$ and $q$ are integers. Note that such an assumption is only valid for surfacebased local extraction of global factor and does not apply to global simulation involving volume domain as in [4]. Recalling the continuity relation (12), (6) becomes

$$
\begin{gathered}
\psi(\bar{r})+\int_{L^{2}} d r^{\prime}\left[\left(-\frac{\partial G_{1}^{P}\left(\bar{r}, \bar{r}^{\prime}\right)}{\partial \bar{n}^{\prime}}+(1-\gamma) v\left(\bar{r}^{\prime}\right) G_{1}^{P}\left(\bar{r}, \bar{r}^{\prime}\right)\right) \psi\left(\bar{r}^{\prime}\right)\right. \\
\left.+\gamma G_{1}^{P}\left(\bar{r}, \bar{r}^{\prime}\right) u\left(\bar{r}^{\prime}\right)\right]=\psi_{i n}(\bar{r}) \\
\psi(\bar{r})+\int_{L^{2}} d r^{\prime}\left[\frac{\partial G_{2}^{P}\left(\bar{r}, \bar{r}^{\prime}\right)}{\partial \bar{n}^{\prime}} \psi\left(\bar{r}^{\prime}\right)-G_{2}^{P}\left(\bar{r}, \bar{r}^{\prime}\right) u\left(\bar{r}^{\prime}\right)\right]=0
\end{gathered}
$$

where $L^{2}$ denotes the investigation patch with an area of $L \times L$, $\psi=\psi_{1}=\psi_{2}$, and $u=\sqrt{1+f_{x}^{2}+f_{y}^{2}} \hat{n} \cdot \nabla \psi_{2}$ with $f_{x}=\partial f / \partial x$. The periodic Green's function and its normal derivative are

$$
\begin{aligned}
G_{i}^{P} & =\sum_{p=-\infty}^{\infty} \sum_{q=-\infty}^{\infty} \frac{\exp \left(j k_{i}\left|\bar{r}-\bar{r}^{\prime}-\hat{x} p L-\hat{y} q L\right|\right)}{4 \pi\left|\bar{r}-\bar{r}^{\prime}-\hat{x} p L-\hat{y} q L\right|} \\
\frac{\partial G_{i}^{P}\left(\bar{r}, \bar{r}^{\prime}\right)}{\partial \bar{n}^{\prime}} & =\sqrt{1+{f_{x}^{\prime}}^{2}+{f_{y}^{\prime}}^{2}} \hat{n}^{\prime} \cdot \nabla^{\prime} G_{i}^{P} .
\end{aligned}
$$

The periodic Green's function in (14) can be efficiently computed by the Ewald method [19], which requires very few terms to converge.

Unlike the simplifying rectangular patches used in [11], plane triangular patches are adopted here to discretize the rough surface, which is more capable of conforming to rough surface with arbitrary curvatures and, thus, helps to improve the accuracy for large roughness. The IE (13) is solved by MOM with point matching, which results in the following matrix equation:

$$
Z J=J_{\text {in }}
$$

where $Z=\left[\begin{array}{cc}A_{1}+(1-\gamma) B_{1} V & \gamma B_{1} \\ A_{2} & B_{2}\end{array}\right], \quad J=\left[\begin{array}{c}\Psi \\ U\end{array}\right] \quad$ and $J_{i n}=\left[\begin{array}{c}\Psi_{i n} \\ 0\end{array}\right]$. The details of the matrix elements in $A_{i}, B_{i}$, and $V$ are shown in (16)-(18), respectively

$$
\begin{aligned}
& A_{i}(m, n)= \begin{cases}\frac{1}{2}+(-1)^{i} \int_{\Delta s_{m}} d r \frac{\partial G_{i}^{P}\left(\bar{r}_{m}, \bar{r}_{m}\right)}{\partial \bar{n}_{m}}, & m=n \\
(-1)^{i} \frac{\partial G_{i}^{P}\left(\bar{r}_{m}, \bar{r}_{n}\right)}{\partial \bar{n}_{n}} \Delta s_{n}, & m \neq n\end{cases} \\
& B_{i}(m, n)= \begin{cases}(-1)^{i+1} \int_{\Delta s_{m}} d r G_{i}^{P}\left(\bar{r}_{m}, \bar{r}_{m}\right), & m=n \\
(-1)^{i+1} G_{i}^{P}\left(\bar{r}_{m}, \bar{r}_{n}\right) \Delta s_{n}, & m \neq n\end{cases} \\
& V(m, n)= \begin{cases}\mid \hat{n}_{m} \times \hat{n}_{m} \times\left(\nabla \times \hat{t}_{H_{m}}\right),, & m=n \\
0, & m \neq n\end{cases}
\end{aligned}
$$

where $\Delta s_{n}$ is the area of the $n$th triangular patch projected on the mean plane. 
Equation (15) can be efficiently solved in $O(N \log (N))$ complexity, where $N$ is the number of discretized elements, with numerical solvers such as the fast-Fourier-transformbased iterative method [20] and the UV-multilevel partitioning method [21]. Once the scalar surface unknowns $\psi$ and $u$ are solved, the energy of the scalar wave absorbed by the conductor beneath the investigation patch is computed in an analogous form as (11)

$$
\begin{aligned}
P_{r} & =\frac{1}{2} \iiint_{V} \nabla \psi(\bar{r}) \nabla \psi^{*}(\bar{r}) d v \\
& =\frac{1}{2} \iint_{\partial V} \operatorname{Re}\left\{\psi^{*}(\bar{r}) \frac{\partial \psi(\bar{r})}{\partial \hat{n}}\right\} d s \\
& =\frac{1}{2} \iint_{S} \operatorname{Re}\left\{\psi^{*}(\bar{r}) \frac{\partial \psi(\bar{r})}{\partial \hat{n}}\right\} d s \\
& =\frac{1}{2} \iint_{L^{2}} \operatorname{Re}\left\{\psi^{*}(\bar{r}) u(\bar{r})\right\} d r
\end{aligned}
$$

where the second equality results from applying the Green's first identity and (5) and the third equality is due to the fact that $\psi$ vanishes at the bottom surface, and the contributions from opposite side walls cancel out each other under the doubly periodic assumption. For a smooth conducting plane subjected to the same incident wave, we assume the well-known exponential decrease of $\psi$ w.r.t. the penetration depth

$$
\psi(\bar{r})=\psi_{0} \exp \left(k_{2} z\right)
$$

where $\psi_{0}$ is the value of $\psi$ at $z=0$ and, based on (16), should be $2\left|\psi_{\text {in }}\right|$. In view of (19) and (20), the energy loss for the smooth-surface conductor is then given by $P_{s}=L^{2} /(2 \delta)$.

\section{Comparison With Vectorial EM Modeling}

The rigorous surface-integral formulation based on EM theory of rough-surface scattering from penetrable objects can be found in a number of literature, e.g., [22]. The surface is generally discretized by RWG triangular elements, each of which is associated with two vectorial unknowns, usually the electric and magnetic surface-current densities. The size of the resultant matrix equation, if converted to corresponding scalar equations, is roughly $6 N \times 6 N$, where $N$ is the number of discretized triangular patches. If the low-frequency problem is taken into account, the common remedies, e.g., high-order basis function or high-order operator, will significantly increase the computational cost. In contrast, SWM gives a matrix with size of only $2 N \times 2 N$, leading to a significant reduction in solution time and memory storage. Since no hypersingular operator, i.e., second derivative of Green's function, appears in (6), the SWM formulation is free of the involved low-frequency problem and, thus, avoids the excessive complexity introduced by the corresponding remediation. In addition, SWM also enjoys much simpler implementation and less overhead than EM modeling.

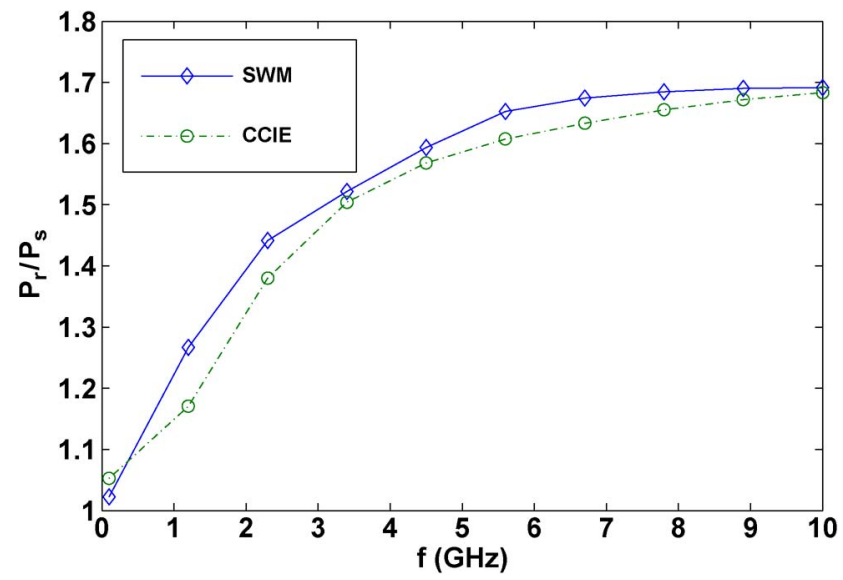

Fig. 4. SWM versus EM model for Gaussian CF $(\sigma=\eta=1 \mu \mathrm{m})$.

TABLE I

CPU TIMES OF SWM AND CCIE

\begin{tabular}{ccccc}
\hline Interval & No. of triangles $(N)$ & SWM $(\mathrm{sec})$ & CCIE $(\mathrm{sec})$ & Speedup \\
\hline$\eta / 6$ & 1800 & 8.12 & 541.44 & $67 \mathrm{X}$ \\
\hline$\eta / 8$ & 3200 & 19.72 & 1382.01 & $70 \mathrm{X}$ \\
\hline$\eta / 10$ & 5000 & 39.55 & 2921.38 & $74 \mathrm{X}$ \\
\hline
\end{tabular}

To validate the usage of SWM to approximate vector wave model, a recently developed EM wave model, called current and charge IE (CCIE) [23], is implemented to compute the loss-scaling factor. CCIE avoids the low-frequency breakdown by using electric- and magnetic-charge densities as unknowns, in addition to the traditional surface current densities, i.e., the unknown vector is $[\epsilon \hat{n} \cdot \bar{E}, \hat{n} \times \bar{E}, \hat{n} \times \bar{H}, \mu \hat{n} \cdot \bar{H}]^{T}$. CCIE also leads to well-balanced systems for a wide frequency range by a dedicated normalization scheme, which renders it a suitable verification benchmark for SWM. The system of equations are established based on the electric field integral equation (EFIE) and magnetic field integral equation (MFIE) using the periodic Green's function. The vector surface-current densities $(\hat{n} \times \bar{E}, \hat{n} \times \bar{H})$ are expanded with the RWG basis functions, and the scalar charge densities $(\epsilon \hat{n} \cdot \bar{E}, \mu \hat{n} \cdot \bar{H})$ use piecewise constant-basis functions. Once the surface-current densities are solved, the ohmic loss in the conductor is computed by

$$
\frac{1}{2} \iint_{L^{2}} \operatorname{Re}\left\{\frac{\hat{n} \times \bar{E}(\bar{r}) \cdot\left(\hat{n} \times \bar{H}^{*}(\bar{r}) \times \hat{n}\right)}{\sqrt{f_{x}^{2}+f_{y}^{2}+1}}\right\} d r .
$$

In numerical experiments, SWM and CCIE are both employed to compute the frequency-dependent loss-scaling factors for an individual rough surface generated with Gaussian CF $(\sigma=\eta=1 \mu \mathrm{m})$ and discretized by triangular elements with interval of $\eta / 8$. For a fair comparison, both SWM and CCIE use direct solver for the solution of matrix equations. As seen in Fig. 4, SWM provides a fairly reasonable approximation to the results from vector-wave modeling. The CPU times of SWM and CCIE are shown in Table I with different problem sizes. It can be seen that SWM is more than $60 \times$ faster than CCIE, which can be attributed to its much smaller problem size and less overhead. 


\section{Spectral SSCM}

Equation (15) is solved only for a deterministic surface, i.e., the surface heights and surface derivatives must be explicitly given. If the surface roughness is modeled by a stochastic process described in Section II, (15) becomes a stochastic IE w.r.t. the $N$ correlated random variables of surface height

$$
Z(\bar{h}) J(\bar{h})=J_{i n}(\bar{h}), \quad \bar{h}=\left(h_{1}, h_{2}, \ldots, h_{N}\right) .
$$

MC simulation is widely used to solve (22) and, thus, the rough-surface loss, by randomly sampling the solution space. However, the number of samples might be prohibitively large to guarantee convergence, e.g., 5000 sampling points may be needed for convergence to within $1 \%$. An efficient solver for stochastic equation like (22) has been proposed in [4] that computes the statistical model of surface unknown $J$ via a modified polynomial-chaos expansion and Neumann expansion. Nevertheless, introduction of stochastic expansion to individual matrix elements in an equivalent sense modifies the integral kernel in (13), which, in consequence, may destroy the inherent structure of the system matrix and prevent the potential numerical-acceleration techniques working exclusively for ordinary systems. Moreover, as the calculation of rough-surface loss involves internal interaction between the subvectors of $J$ [cf. (19)], it is not straightforward to recover the statistical model of final rough-surface loss from that of $J$, as the statistics of the product of two correlated stochastic processes is much more complicated than that of a single one.

In view of the aforementioned difficulties and the fact that there are efficient ways to solve the regular IE for each deterministic surface sample, we choose to apply the spectral SSCM [12] to compute the statistical model of rough-surface loss. SSCM is free of the aforementioned difficulties owing to its "sampling" nature, which does not alter the basic solution mechanism in each individual solution [24]. The essence of SSCM is to model the solution of stochastic IE as the homogeneous chaos (HC) expansion

$$
P_{r}(\bar{\xi}) \approx \sum_{|i|=0}^{k} a_{i_{1}, \ldots, i_{D}} H_{i_{1}, \ldots, i_{D}}(\bar{\xi})
$$

where $\bar{\xi}$ is a set of independent random variables of $D$-length decorrelated from the original surface heights $\bar{h}$ by the Karhunen-Loeve $(\mathrm{K}-\mathrm{L})$ expansion. The stopping criteria of $\mathrm{K}-\mathrm{L}$ expansion is that the ratio between the sum of the first $D$ largest eigenvalues and that of all the eigenvalues of the $\mathrm{CF}$ is larger than a user-defined value $r$, i.e., $\sum_{d=1}^{D} \lambda_{d} / \sum_{d=1}^{N} \lambda_{d} \geq r$. Here, $H_{i_{1}, \ldots, i_{D}}(\bar{\xi})$ is a $D$-variable polynomial chaos constructed according to the PDF of $\bar{h}$ [25], which, for Gaussian PDF, is a Hermite polynomial, and $|i|=i_{1}+\cdots+i_{D}$. Note that in (23) $k=1$ corresponds to the first-order modeling, and $k=2$ refers to the second-order modeling which is sufficient for most physical processes. The coefficients of the HC expansion are determined by the $D$-dimensional infinite integration

$$
a_{i_{1}, \ldots, i_{D}}=\int_{-\infty}^{+\infty} d^{D} \bar{\xi} P_{r}(\bar{\xi}) H_{i_{1}, \ldots, i_{D}}(\bar{\xi}) \operatorname{PDF}(\bar{\xi})
$$

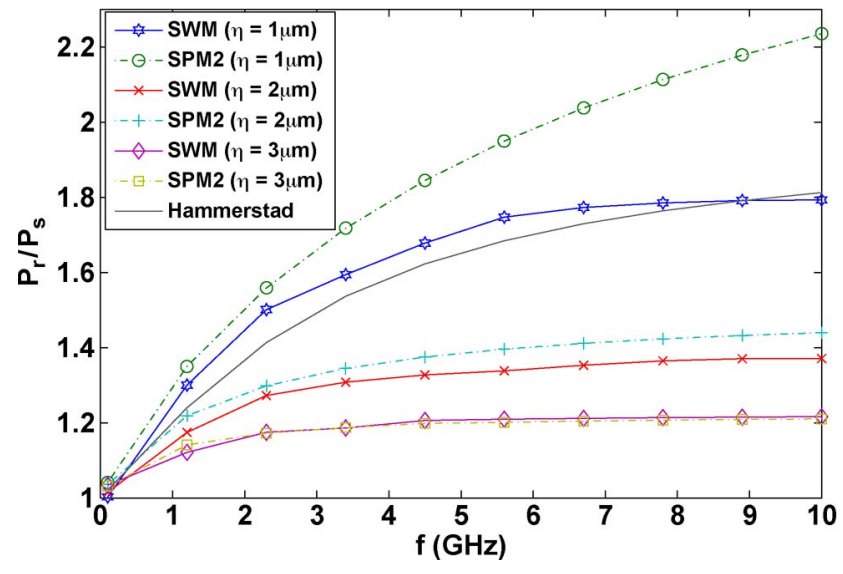

Fig. 5. SWM versus SPM2 and Hammerstad formula for Gaussian CF $(\sigma=1 \mu \mathrm{m}, \eta=1,2,3 \mu \mathrm{m})$.

which can be efficiently computed by the sparse-grid (SG) quadrature [26]. Once the $\mathrm{HC}$ coefficients are determined, the common statistical quantities, mean and variance, of $P_{r}$ can be easily calculated by

$$
\begin{aligned}
\bar{P}_{r} & =a_{0, \ldots, 0} \\
\operatorname{Var}\left(P_{r}\right) & =\sum_{|i|=1}^{k} a_{i_{1}, \ldots, i_{D}}^{2}\left\langle\left(H_{i_{1}, \ldots, i_{D}}\right)^{2}\right\rangle
\end{aligned}
$$

where $\left\langle\left(H_{i_{1}, \ldots, i_{D}}\right)^{2}\right\rangle=\int_{-\infty}^{+\infty} d^{M} \bar{\xi} H_{i_{1}, \ldots, i_{D}}(\bar{\xi}) \operatorname{PDF}(\bar{\xi})$. The major computational cost of SSCM lies in the solution of (15) for the sampling points selected by SG quadrature, to which the aforementioned efficient linear solvers are applicable. Compared with MC simulation, SSCM can achieve a second-order accuracy with much fewer sampling points.

\section{NuMERICAL RESUltS}

In the following numerical experiments, we assume a conductor with resistivity $\rho=1.67 \mu \Omega \cdot \mathrm{cm}$ and a dielectric with relative permittivity 3.7 of common silicon dioxide $\left(\epsilon_{1}=\right.$ $\left.3.7 \times 8.854 \times 10^{-12}\right)$. The periodic length $L$ is $5 \eta$, and the discretized interval is $\eta / 8$. The loss-scaling factors used for comparisons with closed-form models are their mean values computed by SSCM using $r=0.95$ in $\mathrm{K}-\mathrm{L}$ expansion. All programming and simulation were done with Matlab on a 2.4-GHz 2-Gb-RAM PC.

\section{A. Comparison With SPM2}

We first compare SWM with SPM2 which is known to be accurate for small roughness while invalid for large roughness. In Fig. 5, results of SWM and SPM2 are shown for Gaussian CF with various levels of roughness, i.e., $\sigma$ is fixed at $1 \mu \mathrm{m}$, while $\eta$ varies from 1 to $3 \mu \mathrm{m}$. For the smoothest case $(\eta=3 \mu \mathrm{m})$, the two methods are in almost perfect match. The deviation, however, gradually grows as the degree of roughness increases and becomes significant for the roughest case of $\eta=1 \mu \mathrm{m}$. We 


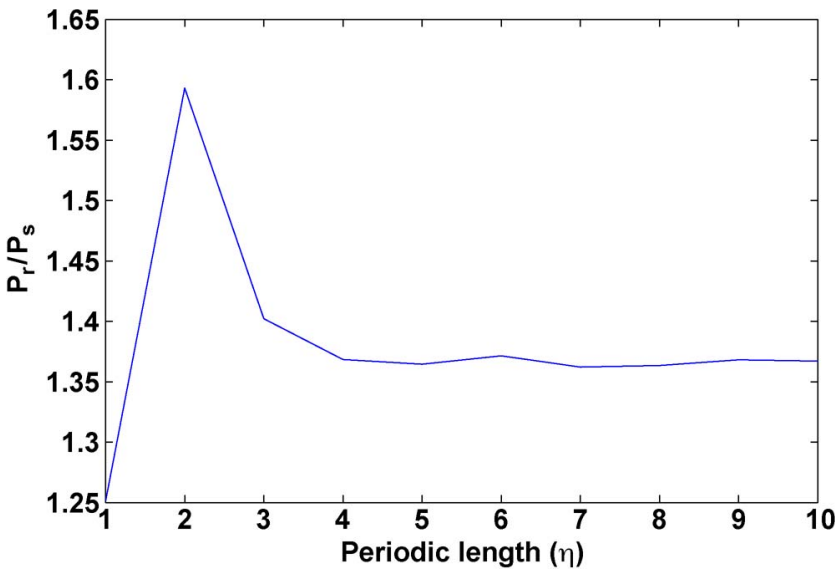

Fig. 6. Influence of the choice of periodic length (Gaussian $\mathrm{CF}$ with $\sigma=1 \mu \mathrm{m}, \eta=2 \mu \mathrm{m})$.

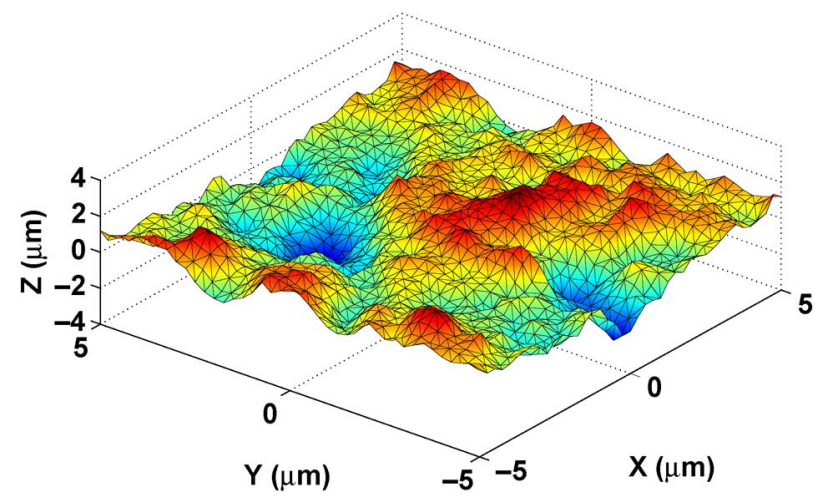

Fig. 7. Simulated rough surface with the extracted CF (26).

also plot the results from the empirical Hammerstad formula (1). As expected, (1) cannot differentiate these distinct cases as they all have the same $\sigma$. The effect of using different periodic lengths is shown in Fig. 6 with Gaussian CF, which justifies the selection of $L$.

We further compare SWM with SPM2 using the CF

$$
C(d)=\sigma^{2} \exp \left\{-\frac{d}{\eta_{1}}\left[1-\exp \left(-\frac{d}{\eta_{2}}\right)\right]\right\}
$$

extracted from practical measurement [13]. A surface generated with CF (26), which is illustrated in Fig. 7, is also continuous as $C^{\prime}(0)=0$, which implies that the surface will turn out to be smooth if sufficiently zoomed in. The partial derivatives of the surface also exist due to the existence of the secondorder derivatives of $C(d)$ at $d=0$ [27]. It has been shown in [13] and [28] that under this roughness setting, SPM2 can give an accurate prediction of surface-roughness loss. The testing frequency is from $100 \mathrm{MHz}$ to $10 \mathrm{GHz}$. As shown in Fig. 8, there is a good agreement between SWM and SPM2. The aforementioned two experiments suggest that SWM is accurate for small roughness cases. Note that HBM is not applicable for these scenarios as the skin depth generally exceeds the height of surface roughness.

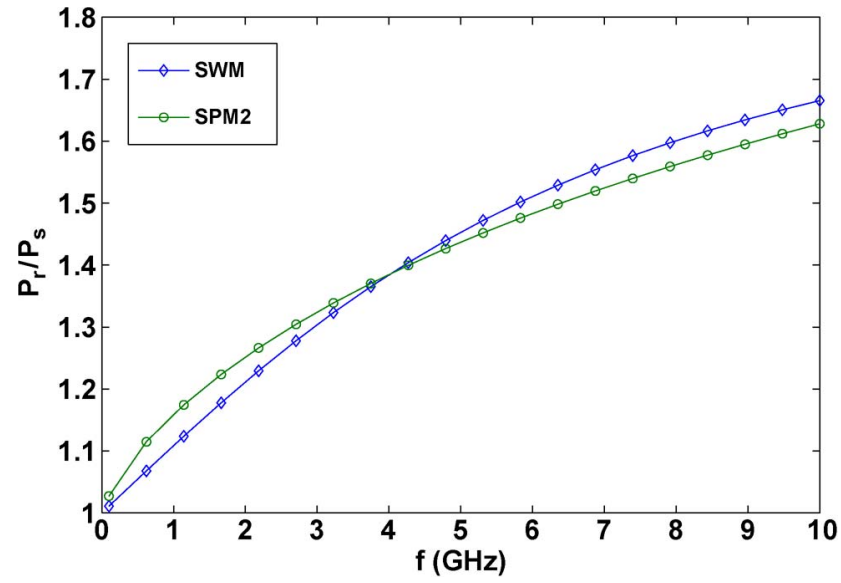

Fig. 8. SWM versus SPM2 with extracted CF $\left(\sigma=0.85 \mu \mathrm{m}, \eta_{1}=1.4 \mu \mathrm{m}\right.$, $\left.\eta_{2}=0.53 \mu \mathrm{m}\right)$.

To demonstrate the inapplicability of SPM2 for roughness with large slope, the loss-scaling factor is plotted w.r.t. varying $\sigma$ ranging from 1 to $3 \mu \mathrm{m}$ and varying the frequency ranging from 1 to $10 \mathrm{GHz}$. The Gaussian CF is used with the correlation length $\eta$ fixed at $2 \mu \mathrm{m}$. Fig. 9(a) shows that for large roughness (the right upper corner), the loss-scaling factors calculated by SPM2 can go beyond three, which is physically impractical. In contrast, Fig. 9(b) shows that SWM can correctly predict the saturation of loss-scaling factor for Gaussian rough surfaces.

\section{B. Comparison With HBM}

In order to verify the effectiveness of SWM under large roughness, we use the results from HBM as the benchmark, whose accuracy has been confirmed by practical measurements for a specific setting in high frequency [7]. Since the CF of randomly distributed hemispherical bosses is not easy to extract, we only compute the loss-scaling factor of a single deterministic half-spheroid located on a conducting plane with the parameters specified in [7]. The rms base radius $b$ of the half-spheroid, which is not explicitly given in [7], is set to be $2.45 \mu \mathrm{m}$. The triangular mesh of the half-spheroid and the remaining plane is shown in Fig. 10. The testing frequency ranges from 1 to $20 \mathrm{GHz}$ in order to keep the skin depth small compared with the height of protrusion. Two types of discretization schemes, rectangular and triangular, are used with the same discretized interval set to be $\delta / 5$ to model the rapid variation of the field inside the conductor. Simulation results are shown in Fig. 11. It can be observed that the two SWM curves track the trend of HBM and saturate at sufficiently high frequencies. The accuracy of the triangular discretization is better than that of the rectangular one while at the cost of doubling the number of unknowns. This comparison demonstrates that SWM remains valid for cases with large roughness and high frequencies. Despite the fact that a direct comparison with SPM2 is unavailable due to the difficulty in extracting spectral-density function, the validity of SPM2, in this case, is dubious as the rms height and surface slope are both large. 


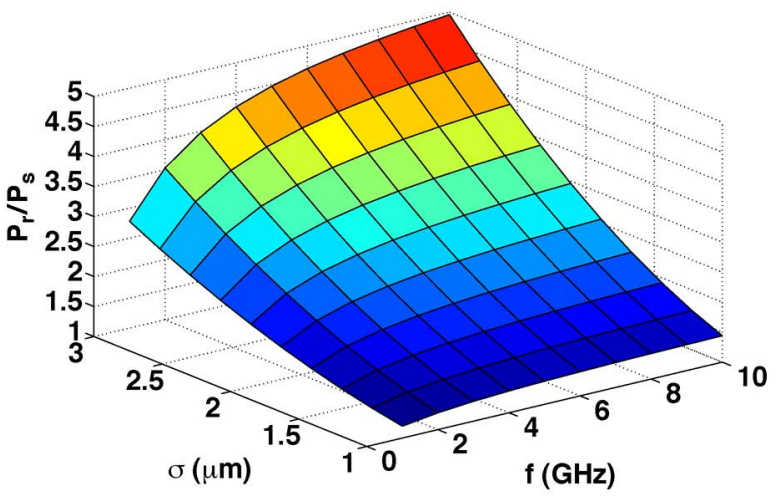

(a)



(b)

Fig. 9. Loss-scaling factor w.r.t. roughness $(\sigma=1 \sim 3 \mu \mathrm{m})$ and frequency $(f=1 \sim 10 \mathrm{GHz})$ for Gaussian CF $(\eta=2 \mu \mathrm{m})$. (a) SPM2. (b) SWM.

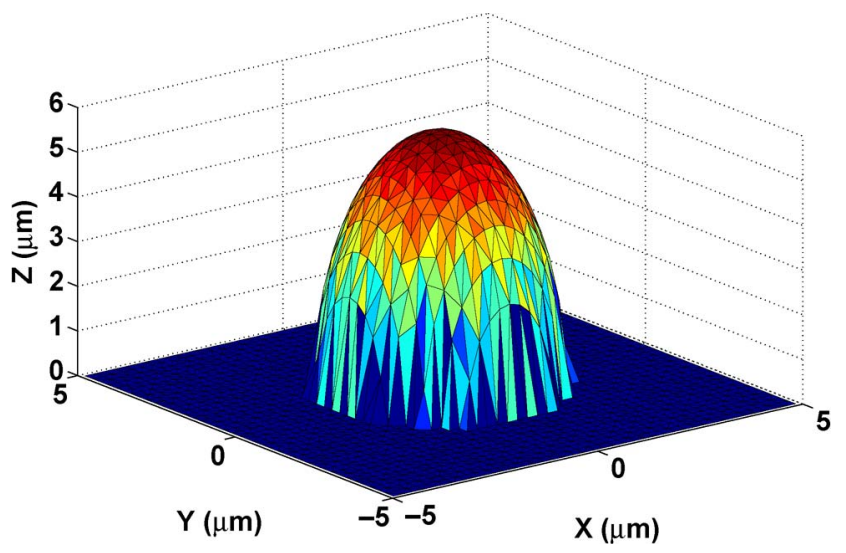

Fig. 10. Single half-spheroid located on a conducting plane $(h=5.8 \mu \mathrm{m}$, $L=9.4 \mu \mathrm{m}, b=2.45 \mu \mathrm{m})$.

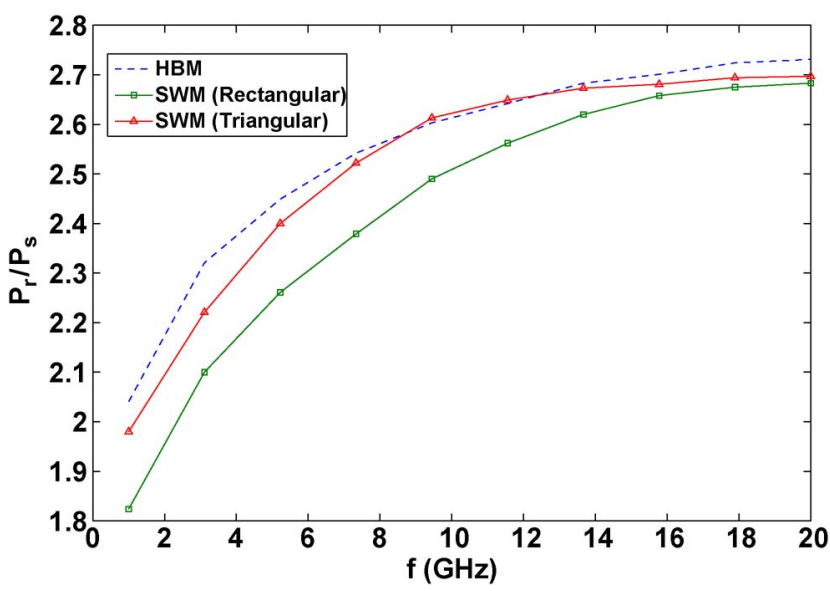

Fig. 11. SWM versus HBM for conducting half-spheroid $(h=5.8 \mu \mathrm{m}$, $L=9.4 \mu \mathrm{m}, b=2.45 \mu \mathrm{m})$.

Again, to demonstrate the inapplicability of HBM for small roughness/low frequencies, the loss-scaling factor is plotted w.r.t. varying $\sigma$ ranging from 1 to $3 \mu \mathrm{m}$ and varying frequency ranging from 0.1 to $1 \mathrm{GHz}$. Single half-spheroid modeling is used with $L=9.4 \mu \mathrm{m}$ and $b=2.45 \mu \mathrm{m}$. Fig. 12(a) shows that for small roughness/low frequencies, HBM predicts erroneous loss-scaling factors that are less than 1 and should be replaced by other closed-form models, e.g., the Hammerstad formula [7]. In contrast, Fig. 12(b) shows that the loss-scaling factor calculated by SWM are all larger than 1 . It is worth noting that the major factors that limit the validity of SPM2 and HBM have different emphases. The former is largely related to the geometrical properties of the roughness under investigation, while the latter relies more on the frequency range that the simulation is conducted. Similar conclusion can be drawn for other analytical techniques. Therefore, it is not a trivial task to select the most appropriate models for a given problem, particularly when generic wideband simulation is considered.

One important advantage of SWM is that it can be used for fast verification of the accuracy of closed-form techniques under specific scenarios. A good example is that, in HBM, a spheroid is actually transformed into an equivalent hemisphere with the same volume [7], since the analytical solution is only available for a hemisphere. Such transformation, however, will inevitably incur an accuracy loss. Fig. 13 shows the lossscaling ratios calculated by SWM and HBM for three halfspheroids with different height-to-base ratios but the same volume $\pi L^{3} / 128$. It can be seen that HBM treats the three cases as the same, whereas SWM differentiates them correctly. This suggests that HBM is more appropriate for roughness with even shapes and may fail for roughness with either very large or small height-to-base ratios. This example also demonstrates the usefulness of an efficient numerical-simulation methodology in verifying the accuracy of closed-form techniques without resorting to expensive experimental measurements.

As reported in [6], the loss-scaling factor for a 3-D rough surface is much higher than that of a 2-D one with the same degree of roughness. Fig. 14 compares the 3-D SWM with a simplified 2-D SWM formulation where the surface height is uniform along $y$-axis. The results also confirm a significant increase of loss with 3-D roughness over 2-D roughness, thereby justifying the necessity of 3-D consideration for roughness analysis.

\section{Stochastic Analysis With SSCM}

To verify the accuracy of the statistical model constructed by SSCM, Fig. 15 shows the cumulative distribution function 


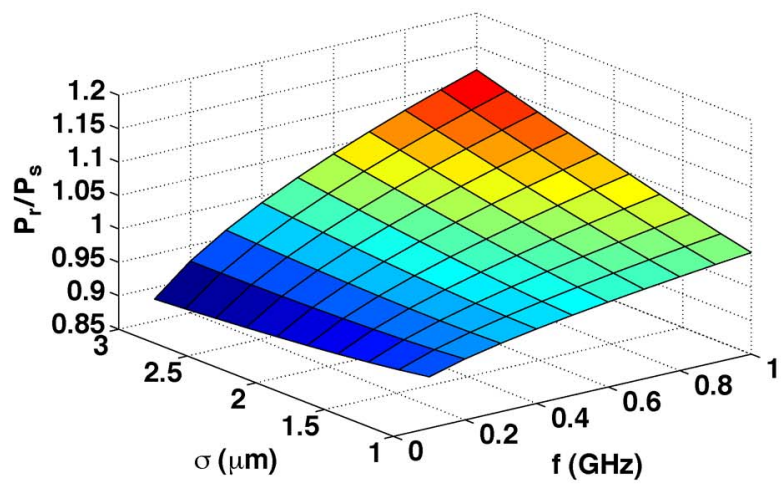

(a)

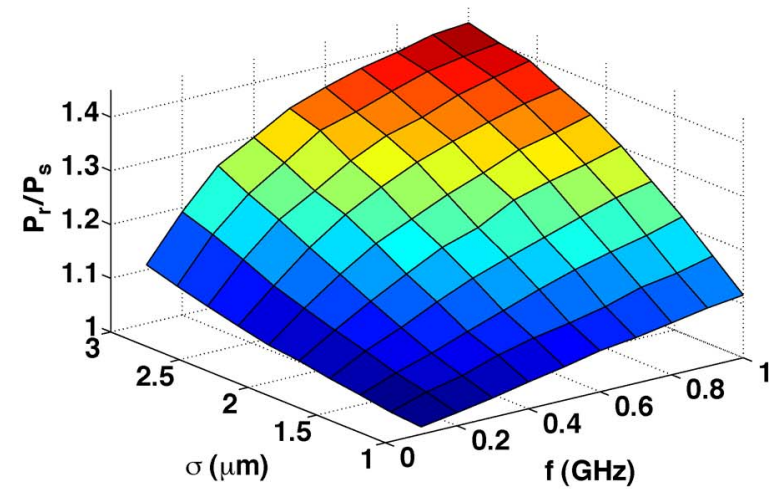

(b)

Fig. 12. Loss-scaling factor w.r.t. roughness $(\sigma=1 \sim 3 \mu \mathrm{m})$ and frequency $(f=0.1 \sim 1 \mathrm{GHz})$ for half-spheroid modeling $(L=9.4 \mu \mathrm{m}, b=2.45 \mu \mathrm{m})$.

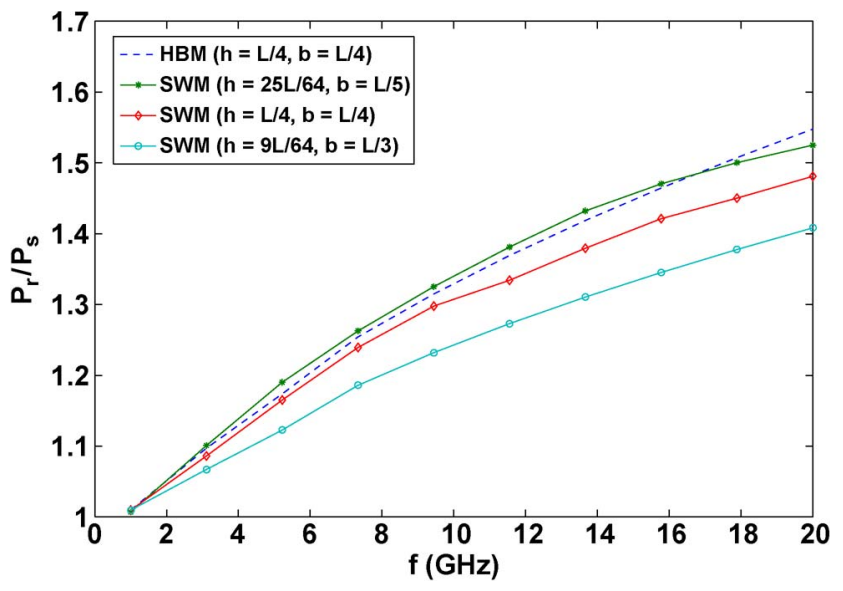

Fig. 13. Power-loss ratios for half spheroids with different $h$ and $b$ $(L=9.4 \mu \mathrm{m})$.

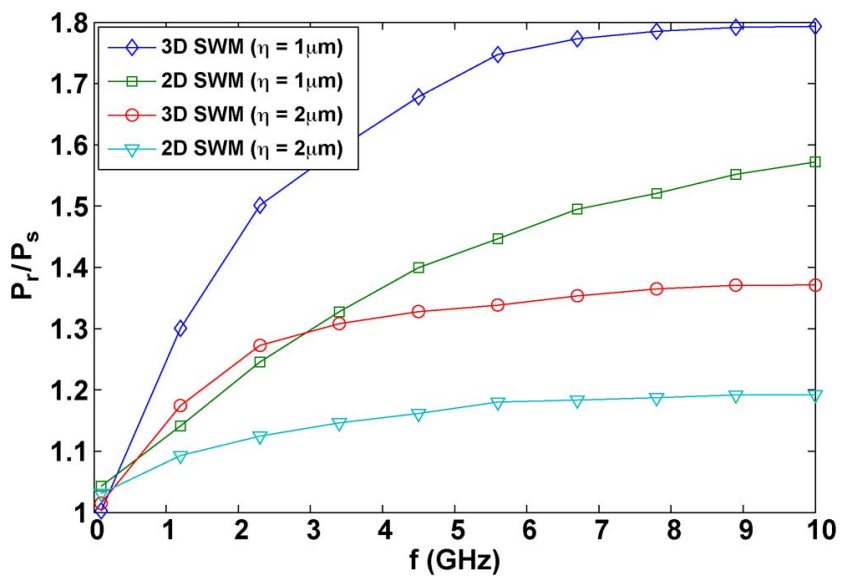

Fig. 14. 3-D SWM versus 2-D SWM for Gaussian CF $(\sigma=1 \mu \mathrm{m}$, $\eta=1,2 \mu \mathrm{m})$.

(CDF) of the loss-scaling factor calculated by first- and secondorder SSCM. The surface is of Gaussian CF with $\sigma=1 \mu \mathrm{m}$, $\eta=2 \mu \mathrm{m}$, and $f=5 \mathrm{GHz}$. Results from MC simulation with 5000 sampling points are used as the benchmark. It can be seen that second-order SSCM is necessary and sufficient to model the distribution of loss-scaling factor under random

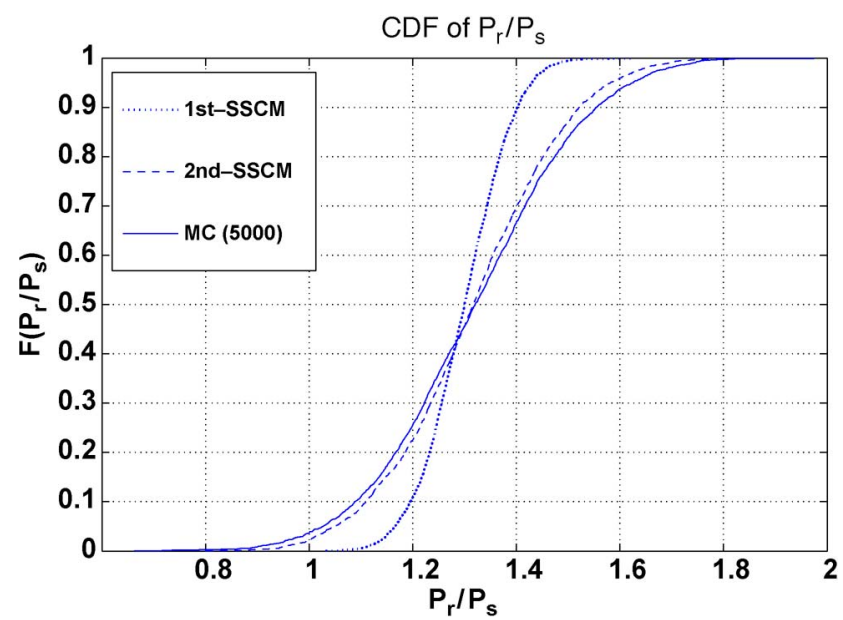

Fig. 15. CDF of $P_{r} / P_{s}(\sigma=1 \mu \mathrm{m}, \eta=2 \mu \mathrm{m}, f=5 \mathrm{GHz})$.

TABLE II

MeAn of Loss-SCALING FACTOR COMPUTEd By MC AND SSCM $(\sigma=1 \mu \mathrm{m}$ AND $f=5 \mathrm{GHz})$

\begin{tabular}{cccc}
\hline \multirow{2}{*}{$\eta$} & \multicolumn{2}{c}{$P_{r} / P_{s}$} \\
& MC & 1st-order SSCM & 2nd-order SSCM \\
\hline $1 \mu m$ & 1.711 & 1.689 & 1.701 \\
\hline $2 \mu m$ & 1.321 & 1.297 & 1.319 \\
\hline $3 \mu m$ & 1.188 & 1.163 & 1.187 \\
\hline
\end{tabular}

TABLE III

VARIANCE OF LOSS-SCALING FACTOR COMPUTED BY MC AND SSCM $(\sigma=1 \mu \mathrm{m}$ AND $f=5 \mathrm{GHz})$

\begin{tabular}{cccc}
\hline \multirow{2}{*}{$\begin{array}{c}\text { Mar }\left(P_{r} / P_{s}\right) \\
\end{array}$} & MC & 1st-order SSCM & 2nd-order SSCM \\
\hline $1 \mu m$ & 0.12 & 0.07 & 0.11 \\
\hline $2 \mu m$ & 0.18 & 0.08 & 0.16 \\
\hline $3 \mu m$ & 0.25 & 0.09 & 0.22 \\
\hline
\end{tabular}

surface roughness. Tables II and III give the corresponding mean and variance of loss-scaling factors for rough surfaces with different $\eta$ 's, which also demonstrates the improvement of accuracy resulting from second-order SSCM. The number of sampling points of SSCM w.r.t. to different accuracy in $\mathrm{K}-\mathrm{L}$ expansion is tabulated in Table IV for Gaussian CF $(\sigma=$ $1 \mu \mathrm{m}, \eta=2 \mu \mathrm{m})$. It shows that SSCM can offer a significant reduction in the amount of sampling points compared with the MC simulation. 
TABLE IV

NuMBER OF SAMPLING POINTS OF SSCM

(GAUSSIAN CF WITH $\sigma=1 \mu \mathrm{m}, \eta=2 \mu \mathrm{m}$ )

\begin{tabular}{ccccc}
\hline$r$ & $D$ & 1st-order SSCM & 2nd-order SSCM & MC \\
\hline $85 \%$ & 18 & 37 & 672 & 5000 \\
\hline $90 \%$ & 22 & 45 & 998 & 5000 \\
\hline $95 \%$ & 28 & 57 & 1605 & 5000 \\
\hline
\end{tabular}

Several remarks are in order:

1) The proposed SWM approach significantly expands the valid range compared with existing analytical techniques like SPM2 and HBM and, thus, enables a generic simulation for different surface roughness over a wide frequency range. It also offers an efficient and convenient way to test the validity of other closed-form techniques without resorting to costly experimental verification.

2) Compared with rigorous EM wave modeling, SWM leads to remarkable computational savings by reducing the number of unknowns and using low-order basis function. In addition, SWM avoids the low-frequency problem that is difficult to handle in EM modeling and, thus, the subsequent expense on correction measures.

3) Complete statistical model of loss-scaling factor beneficial for further stochastic processing is available with SWM. The increased computational cost from randomsurface modeling has been well alleviated by the application of SSCM, by which, much fewer sampling points than $\mathrm{MC}$ simulation are needed to achieve a satisfactory second-order accuracy.

4) For rough surface with exponential $\mathrm{CF}$, the major difficulty in simulation arises from the gradient discontinuity of $C_{e}(d)=\sigma^{2} \exp (-|d| / \eta)$ at $d=0$, which indicates that the surface includes short-length variations down to an arbitrarily small scale, and, as a result, the surface derivatives do not exist. However, in practice, the observation of fine-scale roughness is restricted by the limit of measurement tools and the required modeling accuracy. In this sense, an exponential surface can be viewed as "piecewise continuous" between any two adjacent sampling points provided that the inherent exponential statistics of the surface is properly captured by such sampling, which suggests a viable solution to modeling exponential roughness, e.g., [29].

\section{CONClusion}

The main contribution of this paper has been the development of a generic and efficient methodology SWM for the simulation of rough-surface loss in interconnects and packagings. The key idea is to approximate the complicated EM wave problem with a simplified scalar-wave problem. The major merits of SWM are twofold. First, the significantly enhanced simulation capacity and flexibility eliminate the need of undesirable model selections and model switches and the need of expensive experiments for verifying closed-form techniques. Second, the availability of statistical model in terms of independent random variables is achieved efficiently using SSCM, which in turn enables further stochastic analyses. The excellent applicability of SWM has been verified by benchmarking against a rigorous EM implementation as well as existing closed-form techniques, namely, SPM2 and HBM, in their respective valid regions.

\section{APPENDIX}

The derivation of (9) is given as follows:

$$
\begin{aligned}
\bar{E}_{t}= & -\hat{n} \times(\hat{n} \times \bar{E}) \\
= & -\hat{n} \times\left[-\frac{1}{j \omega \varepsilon} \hat{n} \times\left(\nabla \times\left(\hat{t}_{H} \psi\right)\right)\right] \\
= & \frac{1}{j \omega \varepsilon} \hat{n} \times\left[\hat{n} \times\left(\psi \nabla \times \hat{t}_{H}+\nabla \psi \times \hat{t}_{H}\right)\right] \\
= & \frac{1}{j \omega \varepsilon} \hat{n} \times\left\{\psi \hat{n} \times\left(\nabla \times \hat{t}_{H}\right)\right. \\
& \left.\quad+\left[\nabla \psi\left(\hat{n} \cdot \hat{t}_{H}\right)-\hat{t}_{H}(\hat{n} \cdot \nabla \psi)\right]\right\} \\
= & \frac{1}{j \omega \varepsilon}\left\{\hat{n} \times \hat{n} \times\left(\nabla \times \hat{t}_{H}\right) \psi-\left(\hat{n} \times \hat{t}_{H}\right)(\hat{n} \cdot \nabla \psi)\right\} \\
= & \frac{1}{j \omega \varepsilon}\left\{\hat{t}_{E}\left|\hat{n} \times \hat{n} \times\left(\nabla \times \hat{t}_{H}\right)\right| \psi-\left(\hat{n} \times \hat{t}_{H}\right)(\hat{n} \cdot \nabla \psi)\right\} \\
= & \hat{t}_{E}\left[\frac{1}{j \omega \varepsilon}(v \psi-\hat{n} \cdot \nabla \psi)\right]
\end{aligned}
$$

where the fourth equality results from the vector triple product and the fifth equality is due to $\hat{n} \cdot \hat{t}_{H}=0$. The components of $\hat{t}_{H}$ are determined by the following relationship:

$$
\left\{\begin{array}{l}
-f_{x} t_{H x}-f_{y} t_{H y}+t_{H z}=0 \\
t_{H x}^{2}+t_{H y}^{2}+t_{H z}^{2}=1 \\
t_{H y}=\tan (\theta) t_{H x}
\end{array}\right.
$$

where $\theta$ denotes the azimuth angle of incident $\bar{H}$ field. $\theta$ can be chosen arbitrarily provided the rough surface is isotropic, and, for simplicity, it can be set as 0 .

\section{ACKNOWLEDGMENT}

The authors would like to thank Prof. W. C. Chew for his valuable comments and suggestions to this paper.

\section{REFERENCES}

[1] H. Tanaka, "Precise measurements of dissipation factor in microwave printed circuit boards," IEEE Trans. Instrum. Meas., vol. 38, no. 2, pp. 509-514, Apr. 1989.

[2] A. Deutsch, C. W. Surovic, R. S. Krabbenhoft, and G. V. Kopcsay, "Prediction of losses caused by roughness of metallization in printed-circuit boards," IEEE Trans. Adv. Packag., vol. 30, no. 2, pp. 279-287, May 2007.

[3] G. Brist, S. Hall, S. Clouser, and L. Tsang, "Non-classical conductor losses due to copper foil roughness and treatment," in Proc. ECWC 10th Conf., IPC Printed Circuits Expo., 2005, p. S19-2-1.

[4] T. El-Moselhy and L. Daniel, "Stochastic integral equation solver for efficient variation-aware interconnect extraction," in Proc. 45th Аппи. Conf. DAC, 2008, pp. 415-420.

[5] E. O. Hammerstad and F. Bekkadal, Microstrip Handbook. Trondheim, Norway: Univ. Trondheim, 2001.

[6] X. Gu, L. Tsang, and H. Braunisch, "Modeling effects of random rough interface on power absorption between dielectric and conductive medium in 3-D problem," IEEE Trans. Microw. Theory Tech., vol. 55, no. 3, pp. 511-517, Mar. 2007.

[7] S. Hall, S. G. Pytel, P. G. Huray, D. Hua, A. Moonshiram, G. A. Brist, and E. Sijercic, "Multigigahertz causal transmission line modeling methodology using a 3-D hemispherical surface roughness approach," IEEE Trans. Microw. Theory Tech., vol. 55, no. 12, pp. 2614-2624, Dec. 2007.

[8] L. Daniel, C. S. Ong, S. C. Low, K. H. Lee, and J. White, "A multiparameter moment-matching model-reduction approach for generating geometrically parameterized interconnect performance models," IEEE Trans. Comput.-Aided Design Integr. Circuits Syst., vol. 23, no. 5, pp. 678-693, May 2004. 
[9] H. Chang and S. S. Sapatnekar, "Statistical timing analysis under spatial correlations," IEEE Trans. Comput.-Aided Design Integr. Circuits Syst., vol. 24, no. 9, pp. 1467-1482, Sep. 2005.

[10] Q. Chen and N. Wong, "Efficient numerical modeling of random rough surface effects for interconnect internal impedance extraction," in Proc. IEEE ASP-Des. Autom. Conf., 2007, pp. 152-157.

[11] Q. Chen and N. Wong, "New simulation methodology of 3D surface roughness loss for interconnects modeling," in Proc. IEEE Int. Conf. DATE, 2009, pp. 1184-1189.

[12] H. Zhu, X. Zeng, W. Cai, J. Xue, and D. Zhou, "A sparse grid based spectral stochastic collocation method for variations-aware capacitance extraction of interconnects under nanometer process technology," in Proc. IEEE Int. Conf. DATE, 2007, pp. 1514-1519.

[13] H. Braunisch, X. Gu, A. Camacho-Bragado, and L. Tsang, "Off-chip rough-metal-surface propagation loss modeling and correlation with measurements," in Proc. Int. Conf. Electron. Compon. Technol., 2007, pp. 785-791.

[14] K. F. Warnick and W. C. Chew, "Numerical simulation methods for rough surface scattering," J. Waves Random Media, vol. 11, no. 1, pp. 1-30, Jan. 2001.

[15] P. Yla-Oijala and M. Taskinen, "Well-conditioned Muller formulation for electromagnetic scattering by dielectric objects," IEEE Trans. Antennas Propag., vol. 53, no. 10, pp. 3316-3323, Oct. 2005.

[16] J. A. Ogilvy, Theory of Wave Scattering From Random Rough Surfaces. New York: Taylor \& Francis, 1991.

[17] L. Tsang, J. A. Kong, K. H. Ding, and C. O. Ao, "Scattering of electromagnetic waves," in Numerical Simulations, vol. 2. New York: Wiley, 2001.

[18] S. Kirkup, The Boundary Element Method in Acoustics, 2007. [Online]. Available: http://www.boundary-element-method.com

[19] S. Oroskar, D. R. Jackson, and D. R. Wilton, "Efficient computation of the 2D periodic Greens function using the Ewald method," J. Comput. Phys., vol. 219, no. 15, pp. 899-911, Dec. 2006.

[20] G. Tsabarya and Y. Agnon, "Wave scattering from a rough surface: Solution by an iterative method," Wave Motion, vol. 44, no. 7/8, pp. 626-648, Aug. 2007.

[21] Z. X. Li, "Wave scattering with the UV multilevel partitioning method: Three-dimensional problem of dielectric rough-surface scattering," Microw. Opt. Technol. Lett., vol. 48, no. 7, pp. 1313-1317, Apr. 2006.

[22] S. Y. Chen, W. C. Chew, J. M. Song, and J. S. Zhao, "Analysis of low frequency scattering from penetrable scatterers," IEEE Trans. Geosci. Remote Sens., vol. 39, no. 4, pp. 726-735, Apr. 2001.

[23] M. Taskinen and P. Yla-Oijala, "Current and charge integral equation formulation," IEEE Trans. Antennas Propag., vol. 54, no. 1, pp. 58-67, Jan. 2006.

[24] W. Y. Zhang, W. J. Yu, Z. Y. Wang, Z. P. Yu, R. Jiang, and J. J. Xiong, "An efficient method for chip-level statistical capacitance extraction considering process variations with spatial correlation," in Proc. Conf. DATE, 2008, pp. 580-585.

[25] D. Xiu and G. E. Karniadakis, "The Wiener-Askey polynomial chaos for stochastic differential equations," SIAM J. Sci. Comput., vol. 24, no. 2, pp. 619-644, 2002.

[26] E. Novak and K. Ritter, "Simple cubature formulas with high polynomial exactness," Constr. Approx., vol. 15, no. 4, pp. 499-522, Dec. 1999.

[27] R. J. Adler, The Geometry of Random Fields. New York: Wiley, 1981.

[28] X. Gu, L. Tsang, and H. Braunisch, "Estimation of roughness-induced power absorption from measured surface profile data," IEEE Microw. Wireless Compon. Lett., vol. 17, no. 7, pp. 486-488, Jul. 2007.

[29] P. Xu and L. Tsang, "Bistatic scattering and emissivities of lossy dielectric surfaces with exponential correlation functions," IEEE Trans. Geosci. Remote Sens., vol. 45, no. 1, pp. 62-72, Jan. 2007.

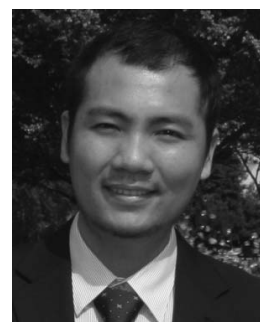

Quan Chen (S'09) received the B.S. degree in electrical engineering from Sun Yat-Sen University, Guangzhou, China, in 2005 and the M.Phil. degree in electronic engineering from The University of Hong Kong, Pokfulam Road, Hong Kong, in 2007, where he is currently working toward the Ph.D. degree in the Department of Electrical and Electronic Engineering, under the supervision of Dr. N. Wong.

His current research interest is in coupled electromagnetic simulation of high-frequency very-largescale integration circuits.

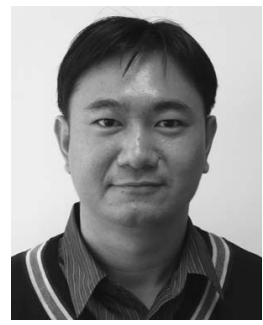

Hoi Wai Choi (M'07-SM'09) received the Ph.D. degree from the National University of Singapore, Singapore, Singapore, in 2002

After postdoctoral training with Prof. M. D. Dawson with the Institute of Photonics, University of Strathclyde, Glasgow, U.K., he has been with the Department of Electrical and Electronic Engineering, The University of Hong Kong, Pokfulam Road, Hong Kong, since 2004, where he is currently an Assistant Professor in the Semiconductor Lighting and Display Laboratory leading a team of researchers investigating frontier research topics including solid-state lighting, GaN microcavities, fiber-coupled microdisplays, 3-D laser micromachining, and device modeling. He has published over 100 papers in leading journals and international conferences, contributed to two book chapters, and has filed for numerous patents.

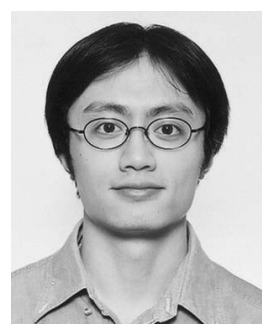

Ngai Wong (S'98-M'02) received the B.Eng. (with first class honors) and Ph.D. degrees in electrical and electronic engineering from The University of Hong Kong, Pokfulam Road, Hong Kong, in 1999 and 2003, respectively.

During 1997-1998, he was an Intern with Motorola Inc., Hong Kong, specializing in product testing. In 2003, he was a Visiting Scholar with Purdue University, West Lafayette, IN. He is currently an Assistant Professor with The University of Hong Kong. His research interests include very large scale integration (VLSI) model order reduction and simulation, digital-filter design, sigma-delta modulators, and optimization problems in communication and VLSI applications. 